\title{
Biases in Ion Transmission Through an Electrospray Ionization-Mass Spectrometry Capillary Inlet
}

\author{
Jason S. Page, Ioan Marginean, Erin S. Baker, Ryan T. Kelly, Keqi Tang, \\ and Richard D. Smith \\ Biological Sciences Division, Pacific Northwest National Laboratory, Richland, Washington, USA
}

\begin{abstract}
A heated capillary inlet for an electrospray ionization mass spectrometry (ESI-MS) interface was compared with shorter versions of the inlet to determine the effects on transmission and ionization efficiencies for low-flow (nano) electrosprays. Five different inlet lengths were studied, ranging from 6.4 to $1.3 \mathrm{~cm}$. As expected, the electrospray current transmission efficiency increased with decreasing capillary length due to reduced losses to the inside walls of the capillary. This increase in transmission efficiency with shorter inlets was coupled with reduced desolvation of electrosprayed droplets. Surprisingly, as the inlet length was decreased, some analytes showed little or no increase in sensitivity, while others showed as much as a 15-fold gain. The variation was shown to be at least partially correlated with analyte mobilities, with the largest gains observed for higher mobility species, but also affected by solution conductivity, flow rate, and inlet temperature. Strategies for maximizing sensitivity while minimizing biases in ion transmission through the heated capillary interface are proposed. (J Am Soc Mass Spectrom 2009, 20, 2265-2272) (C) 2009 Published by Elsevier Inc. on behalf of American Society for Mass Spectrometry
\end{abstract}

$\mathrm{T}$ The need for improved electrospray ionizationmass spectrometry (ESI-MS) sensitivity [1] and quantitation [2] has been the driving force behind many of the recent technological advances in the field $[3,4]$. One of the developments providing the largest impact to these two areas has been the refinement and effective use of lower flow rate electrosprays $[5,6]$, termed nanoESI [7]. Increased sensitivity with nanoESI is attributed to improvements in ESI efficiency caused by the creation of smaller, more highly charged droplets [8]. The size of the droplets generated by cone-jet electrosprays is proportional to the relaxation length $r^{*}=\left(Q \varepsilon \varepsilon_{0} / \kappa\right)^{1 / 3}$, where $Q$ is the liquid flow rate, $\kappa$ is solution conductivity, $\varepsilon$ is relative permittivity, and $\varepsilon_{0}$ is vacuum permittivity [8]. This scaling shows that reduced flow rates result in smaller droplets, which require fewer Rayleigh fission events to liberate gasphase ions. Additionally, the spray current scales as $I \sim$ $(\gamma Q \kappa / \varepsilon)^{1 / 2}$ [8], where $\gamma$ is the liquid surface tension. This indicates that there is a greater charge density on the initial droplets formed at lower flow rates, ensuring a larger number of excess charges per analyte molecule. Transmission efficiency may also improve because the electrospray emitter can be placed closer to the interface inlet, which greatly increases the efficiency to sample and transmit the ions/droplets into the

Address reprint requests to Dr. R. D. Smith, Biological Systems Analysis and Mass Spectrometry Division, Pacific Northwest National Laboratory, P.O. Box 999/MS K8-98, Richland, WA 99352, USA. E-mail: rds@pnl.gov instrument [9]. Improved quantitation from nanoESI has been shown to be the result of decreased charge competition and the ability to better ionize less surface active species in the charged droplets [2].

While the ESI-MS community has trended towards lower flow rate operation with both direct infusion and liquid chromatography (LC)-MS analyses [10-12], the standard inlets of the commercial mass spectrometers, which were designed for the early electrosprays operated at relatively large flow rates, remain largely unchanged. The large droplets generated by conventional electrosprays benefit from relatively long residence times inside the heated capillary [13, 14]. However, these conditions may not be optimum for aerosols generated by low-flow electrosprays. Since charged aerosol transmission into the first vacuum chamber of the mass spectrometer is a dominant factor for overall ESI-MS sensitivity $[15,16]$, it is important to understand how typical atmospheric inlets (i.e., capillary tubes and orifices) affect both transmission and ionization efficiency and how the requirements of these devices can change when a lower flow rate electrospray is used. However, standard MS instrumentation does not allow for straightforward decoupling of the effects of transmission and ionization. Therefore, we recently used a vacuum chamber simulating the front-end of a mass spectrometer to study the effect of flow rate, emitter-toinlet distance, and capillary temperature on the loss and transmission of the charged aerosol through a standard capillary inlet interface [9]. Many of the factors conven- 
tionally associated with improved ionization efficiency were shown to negatively affect the transmission efficiency and vice versa. For example, decreasing the distance between the emitter and inlet improved the transmission efficiency but resulted in lower ionization efficiency (presumably due largely to less effective desolvation). Similarly, higher capillary temperatures improved the ionization efficiency due to improved desolvation, but had a negative effect on the transmission efficiency, (which can be attributed to increased diffusion to the walls, etc). The overall achievable sensitivity of the mass spectrometer depends on both ionization and transmission efficiency, but optimum choices are not clear, particularly when different flow rates or solvent mixtures are used for ESI.

In the present work, we varied the capillary inlet length to elucidate the effects on electrospray current, transmission efficiency, and MS peak intensity, with an aim to determine an optimal inlet geometry for low flow rate ESI-MS. Two different electrospray solvent mixtures were used (a low-conductivity solvent mixture, common for infusion studies, and a high-conductivity solvent mixture, common for reversed-phase LC separations) to optimize inlet length and temperature for different ESI solvent conductivities. The results are then evaluated to characterize the ion losses inside the capillary inlet, better understand the origin of biases in electrospray current transmission, and point the way to further refinement and optimization of ESI interfaces currently in use in MS.

\section{Experimental}

\section{Sample Preparation}

The low-conductivity solvent mixture $(\kappa=0.13 \mathrm{mS} /$ $\mathrm{cm})$, referred to herein as the infusion solvent, was prepared by combining purified water (Barnstead Nanopure Infinity system; Dubuque, IA, USA) and methanol (HPLC grade, Fisher Scientific, Fair Lawn, NJ, USA) in a 1:1 ratio and adding acetic acid (SigmaAldrich, St. Louis, MO, USA) at $1 \%$ vol/vol. The highconductivity solvent mixture $(\kappa=2.28 \mathrm{mS} / \mathrm{cm})$, which we term the LC solvent, was a mixture of $40 \%$ mobile phase B in A. Mobile phases A and B consisted of $0.2 \%$ acetic acid and 0.05\% TFA (both from Sigma-Aldrich) in purified water, and $0.1 \%$ TFA in 1:9 purified water: acetonitrile (Fisher Scientific), respectively. A peptide stock solution contained $10 \mu \mathrm{M}$ bradykinin, neurotensin, angiotensin I, leucine enkephalin, and fibrinopeptide A (all from Sigma-Aldrich) in purified water. This solution was then used to prepare $1 \mu \mathrm{M}$ peptide solutions in the infusion and LC solvents.

\section{ESI Interface Capillary Inlets}

A series of five inlets were constructed with an innerdiameter (i.d.) of $490 \mu \mathrm{m}$ and lengths of 1.3, 2.5, 3.8, 5.1, and $6.4 \mathrm{~cm}$ by silver-soldering sections of stainless steel capillary tubing (part no. 89935K82; McMaster-Carr, Los Angeles, CA, USA) in the center of a brass cylinder body. Threads were machined on the outside of the brass bodies to allow connection to a brass, disc-shaped heating block housed and electrically isolated in the front flange of an ESI interface. The brass heating block was heated using a ceramic ring heater (part no. CER1-02-00,001; Watlow, St. Louis, MO, USA) and regulated by a temperature controller (model CN9000A; Omega, Stanford, CT, USA).

\section{Electrospray Current Measurements in the ESI Interface}

Electrospray current transmission measurements were performed using a custom ESI capillary inlet interface [17] attached to a single quadrupole mass spectrometer (MSD1100; Agilent Technologies, Santa Clara, CA, USA). The temperature of the inlets was $120^{\circ} \mathrm{C}$ unless otherwise indicated, and the pressure in the heated capillary inlet chamber was 2 Torr. The electrospray current lost to the capillary inlet and transmitted through the inlet collected on a $4 \times 4 \mathrm{~cm}$ brass plate downstream of the capillary exit were separately measured by a picoammeter (model no. 6485; Keithley, Cleveland, $\mathrm{OH}, \mathrm{USA}$ ) referenced to ground. All current measurements were an average of 100 consecutive measurements using the built-in data acquisition capabilities of the picoammeter. All measurements were performed in triplicate.

\section{ESI Setup}

An electrospray emitter was manufactured by chemically etching [18] a section of 20- $\mu$ m-i.d./150- $\mu$ m-o.d. fused silica capillary tubing (Polymicro Technologies, Phoenix, AZ, USA). The emitter was connected to a transfer capillary and a $100-\mu \mathrm{L}$ syringe (Hamilton, Las Vegas, NV, USA) by a stainless steel union, which also served as the connection point for the electrospray high voltage using a Bertan high voltage power supply (model 205B-03R; Hicksville, NY, USA). All solutions were infused from a Harvard Apparatus model PHD2000 syringe pump (Holliston, MA, USA). A three-dimensional translation stage (Newport, Irvine, CA) controlled the placement and movement of the electrospray emitter. For all experiments, the emitter distance to the capillary inlet was $1 \mathrm{~mm}$.

\section{MS Instrumentation}

Mass spectra were acquired using a single quadrupole mass spectrometer (Agilent Technologies) over a 50$1000 \mathrm{~m} / \mathrm{z}$ range in positive ESI mode with a $0.1 \mathrm{~m} / \mathrm{z}$ step size. Each spectrum was the result of an eight-scan average and all experiments were repeated in triplicate. The same heated capillary inlets, peptide solutions, emitter, and ESI conditions (voltage, distance, flow rate, 
etc.) used for electrospray current measurements were also used for the MS experiments.

\section{Results and Discussion}

\section{Inlet Length Effects with a High-Conductivity Solvent}

A conventional approach to improve ESI efficiency is to acidify the solution to increase its conductivity, which results in smaller initial droplets and larger spray currents. In addition, ion-pairing agents, such as trifluoroacetic acid (TFA), are often added to LC separations to decrease peak broadening [19], which also increases the solution conductivity. We recently demonstrated that low-flow electrosprays of highly conductive solvents show increased MS signal when operated in the pulsating regime instead of the more preferred cone-jet regime, which produces monodisperse droplets [20]. We correlated this observation with improved transmission efficiencies through the capillary inlet of the mass spectrometer when the pulsating regime was used. This suggested that the capillary inlet has a large influence in ESI-MS performance, especially when low-flow electrosprays are employed, leading us to explore the effects of inlet length on nanoESI current transmission and desolvation.

The effect of capillary inlet length on electrospray current transmission efficiency was investigated by infusing a peptide solution in a typical LC solvent and using a series of capillary inlets having different lengths but the same i.d. Figure 1a shows the amount of electrospray current transmitted through the inlets and detected on a charge collector located inside the capillary inlet vacuum chamber. This experiment was repeated using three different flow rates, and the results show the large influence that the inlet can have on electrospray current losses. For example, a flow rate of 50 $\mathrm{nL} /$ min provides a transmitted current of $23 \mathrm{nA}$ with the $1.3-\mathrm{cm}$-long inlet, which quickly drops off to only $2 \mathrm{nA}$ with the 6.4-cm-long inlet; the trend is similar at the other two flow rates. The corresponding transmission efficiencies, calculated by dividing the transmitted currents from Figure 1a by the total electrospray currents, are shown in Figure $1 \mathrm{~b}$. At these flow rates, the effect on transmission efficiency is similar, dropping from $\sim 18 \%$ to $\sim 2 \%$ between the shortest to the longest capillary inlets. In addition, the largest change in transmission efficiency occurs at the shorter inlets. For example, the efficiency drops from $\sim 18 \%$ to $8 \%$ from the 1.3 to the $2.5 \mathrm{~cm}$ inlet, but it only decreases from $\sim 3 \%$ to $2 \%$ from the 5.0 to the $6.4 \mathrm{~cm}$ inlet. This emphasizes that the front section of the inlet has the largest electrospray current loss, possibly due to high space charge [16] and slow gas acceleration in that area.

To understand the changes in transmission efficiency, the gas conductance for each inlet was calculated using common relationships of gas flow through a narrow tube with respect to i.d., length, and temperature [21]. Varying the inlet length while holding the i.d. constant caused the gas conductance, or throughput, of
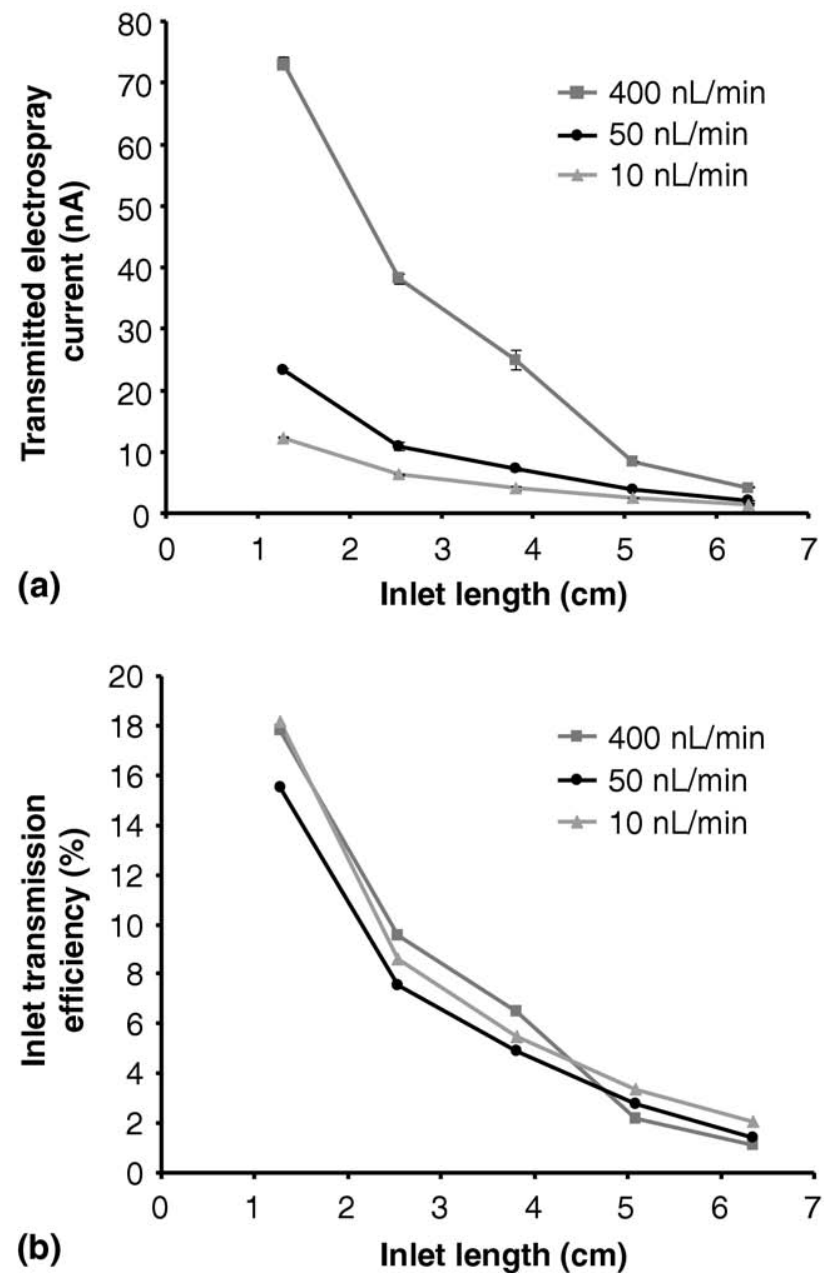

Figure 1. Transmitted electrospray current (a) and electrospray current transmission efficiency (b) versus capillary inlet length for a $1 \mu \mathrm{M}$ peptide solution in a LC solvent mixture at various electrospray flow rates.

the inlet to increase as the length was shortened. For example, the conductance increased from a calculated 0.021 to $0.029 \mathrm{~L} / \mathrm{s}$ between the 6.4 and $3.8 \mathrm{~cm}$ inlets and increased further to $0.054 \mathrm{~L} / \mathrm{s}$ with the $1.3 \mathrm{~cm}$ inlet. The overall increase in gas conductance was 2.6 -fold between the shortest and longest inlets, which is much smaller than the $\sim 10$-fold increase in transmitted current. This indicates that the increase in transmitted current when a shorter inlet is used cannot be attributed to increased gas conductance alone.

Transmitted electrospray current does not directly correlate to instrument sensitivity as the current is a combination of gas-phase ions, clusters/residues, and charged droplets, and the relative contributions of these species are affected by their ability to desolvate in the inlet capillary. To better understand the interplay of the increased transmission efficiency and the decreased desolvation efficiency resulting from the use of shorter inlets, the inlet length experiment was repeated with the charge collector removed from the ESI interface, which allowed the transmitted electrospray current to 
continue to the mass analyzer. Mass spectra were acquired and three representative MS peaks were chosen; $m / z 354$ (bradykinin $3^{+}$), $m / z 559$ (neurotensin $3^{+}$), and $m / z 769$ (fibrinopeptide $\mathrm{A}^{+}$). Figure 2 plots the relative intensity of these ions versus inlet length at three different flow rates. For all three of these ions, decreasing the length increased the intensities. This suggests that the electrospray current transmission efficiency improvement $(\sim 10-$
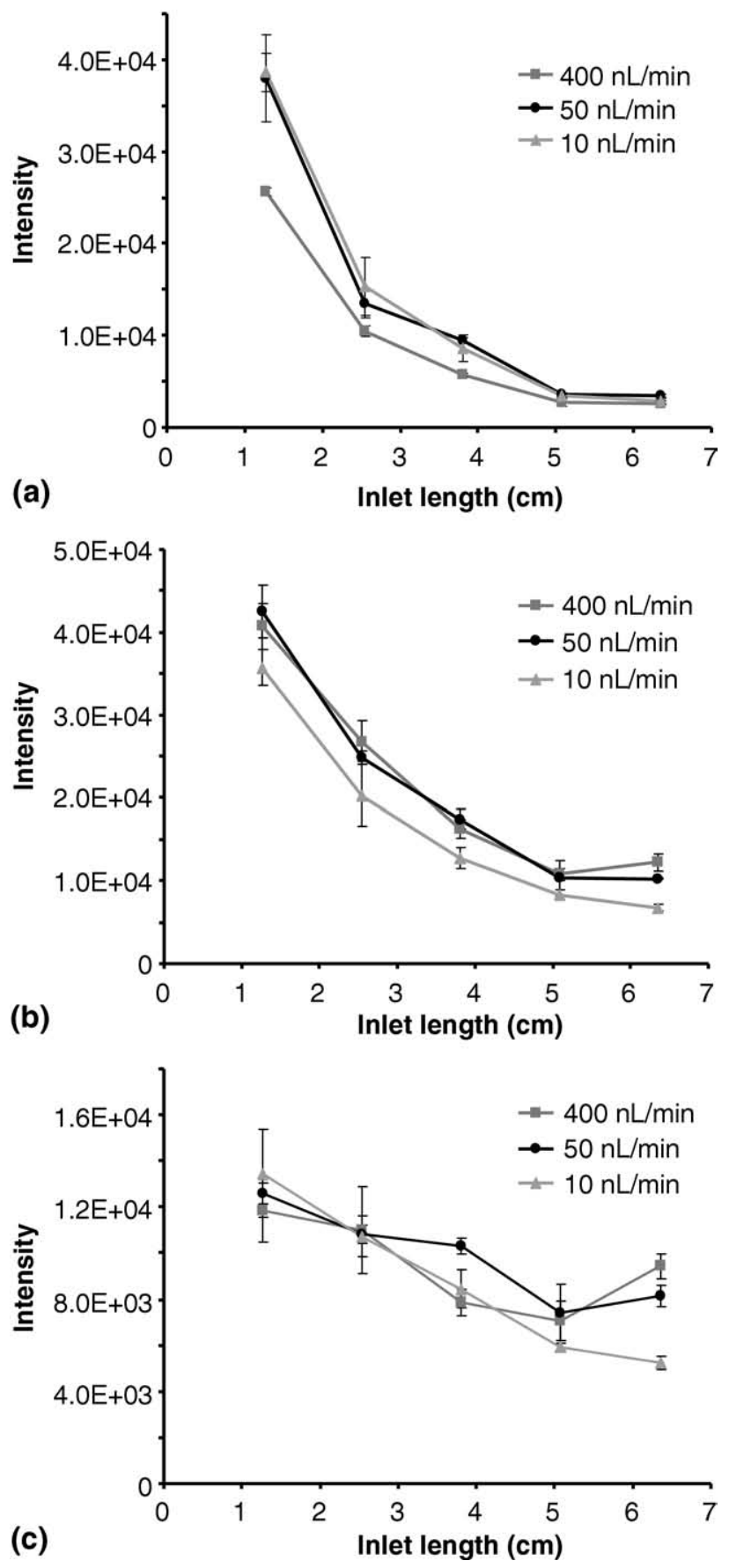

Figure 2. MS intensity versus capillary inlet length for a $1 \mu \mathrm{M}$ peptide solution in a LC solvent mixture at various electrospray flow rates. Three peptides are shown; (a) bradykinin $3^{+}(\mathrm{m} / \mathrm{z}$ 354.3), (b) neurotensin $3^{+}(\mathrm{m} / \mathrm{z} 558.5)$, (c) fibrinopeptide A $2^{+}(\mathrm{m} / \mathrm{z}$ $769.0)$. fold; Figure 1) from using the shorter inlets outweighs the loss in desolvation efficiency due to reduced residence times in the heated capillary. Thus, the overall change in MS signal shows that transmission plays a larger role with the LC solvent than desolvation for these flow rates and inlet temperature (Figure 2).

The data in Figure 2 also show an unpredicted outcome; the rate of intensity change is substantially different for the three peptides. In other words, the increased transmission efficiency from shorter inlets was not universal. Plotting the MS results for the observed enhancement factor (ratio of the intensity from the shortest inlet to the intensity from the longest inlet) versus the flow rate better reveals this variation (Figure 3). The three peptides in this study show substantially different enhancement factors, ranging from $\sim 11$-fold for bradykinin $3^{+}$to $\sim 2$-fold for fibrinopeptide $\mathrm{A}^{+}$, and the enhancement factor is greater at the lowest flow rates. The use of longer inlets resulted in larger ion losses inside the capillary, which became even more pronounced at lower flow rates. This is indicative of space-charge repulsion and diffusion losses inside the inlet. Losses due to space-charge occur since the ions and charged droplets entering the inlet have a net charge density. The charge density causes an expansion of the ion/droplet cloud towards the walls of the metal inlet capillary, which possesses no charge according to Gauss' law, resulting in analyte loss as charged species are neutralized upon impact with the walls. The electric field created by space-charge is greatest at the entrance of the inlet where the charge density is the largest and decreases along the length of the inlet as charge is lost.

To explore why transmission efficiency is reduced by varying degrees for different ions, the mobility of each ion was studied in nitrogen buffer gas with ion mobility spectrometry (IMS)-MS [22]. The mobility determines how quickly ions move under the influence of an electric field and the proportionality constant $(K)$ can be extracted for each ion, with faster ions having higher $K$. In the case of ion and charged droplet transmission through an inlet described here, higher mobility species and larger electric fields are expected to increase the drift velocity of the ions and droplets towards the inlet wall. As seen in eq 1, the mobility coefficient can be related to the physical properties of the charged particle as follows [22]:

$$
K_{o}=\frac{3 q}{16 N} \cdot\left(\frac{2 \pi}{\mu k_{b} T}\right)^{1 / 2} \cdot \frac{1}{\sigma}
$$

where $N$ is the buffer gas number density, $\sigma$ is the cross section, $k_{b}$ is the Boltzmann constant, $T$ is the temperature, $q$ is the charge, and $\mu$ is the reduced mass of the collision partners. Equation 1 also shows that greater net charge and smaller particle cross section lead to increased mobility. As discussed in the introduction, the size and charge of the electrosprayed droplet is dependent on the flow rate. Operating the electrospray at lower flow rates creates smaller droplets having a 


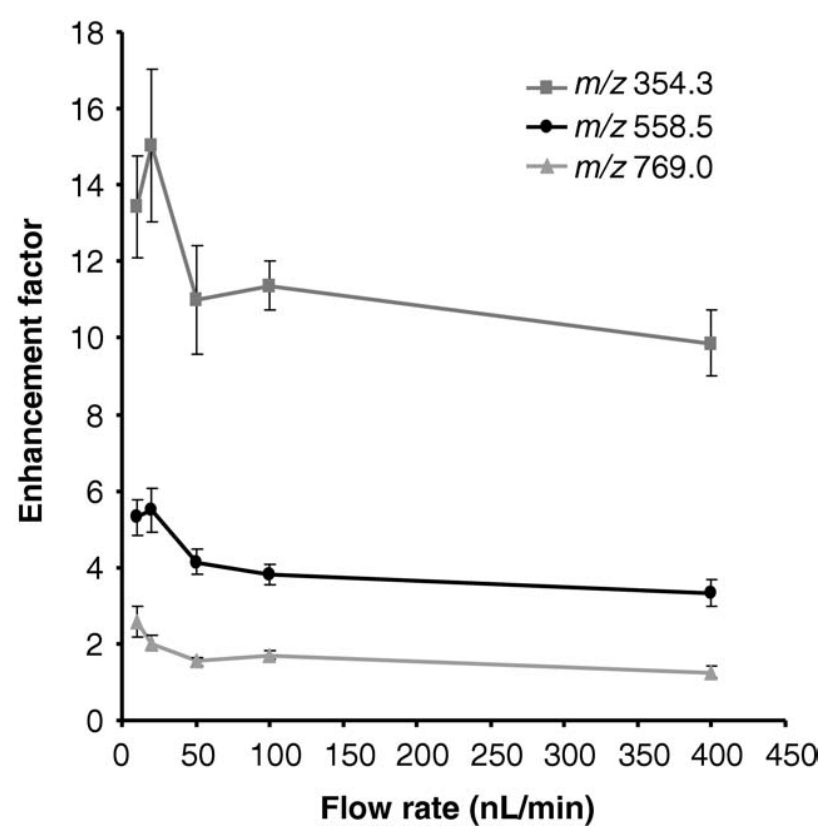

Figure 3. MS signal enhancement factor between the use of a 1.3 $\mathrm{cm}$ inlet and a $6.4 \mathrm{~cm}$ inlet versus flow rate for three different peptides from a $1 \mu \mathrm{M}$ peptide solution in a LC solvent mixture. Enhancement factor is the ratio of the intensity from using the shortest inlet to the intensity from using the longest inlet.

higher charge density, which increases the droplet mobilities. In addition, the smaller droplets liberate free ions more rapidly, which further increases the average mobility of the species in the electrosprayed ion/ droplet cloud. This increases the loss rate for low-flow electrosprays and provides an explanation for the rising trend lines with lower flow rates in Figure 3. The large difference in enhancement factors between the three peptides in Figure 3 can be due to the analytes having different mobilities [23, 24]. Peptides having higher mobilities, such as bradykinin $3^{+}$, should have a greater rate of loss inside the capillary inlet and therefore should suffer disproportional losses through longer inlets, making the factor of intensity increase greater when a shorter inlet is used.

Diffusional inside the inlet can also lead to analyte loss. Fick's law suggests that the ion flux density $(\bar{J})$ is proportional to the diffusion coefficient $(D)$ and the number density gradient $(\bar{\nabla} n)$ :

$$
\bar{J}=-D \bar{\nabla} n
$$

Combining the Einstein-Smoluchowski relation with Stoke's law gives the relationship of the diffusion coefficient with respect to the particle size [25]:

$$
D=\frac{k_{b} T}{6 \pi \eta r}
$$

Where $\eta$ is the viscosity and $r$ is the particle radius. This indicates that diffusion loss in the capillary inlet will be dependent on the size of the droplets/ions, as opposed to space-charge loss, which will be dependent on both size and charge.

Since both space-charge and diffusion losses are expected to occur inside the capillary inlet, the enhancement factors between the shortest and longest inlet were separately plotted with respect to peptide cross section and mobility to differentiate between diffusion and space-charge-driven losses. The mobilities and cross sections for each ion were obtained experimentally (Table 1). Figure 4 plots the enhancement factor versus mobility for all detected peptide peaks from the inlet length study. Peptides having mobilities greater than $\sim 1.1 \mathrm{~cm}^{2} /$ Vs display a rising enhancement factor, corresponding to greater losses in the longest inlet. Interestingly, there appears to be a threshold mobility below which no trend in enhancement factor is observed, and above which the enhancement factor is mobility-dependent. This could be a result of the large differences in ion transmission and desolvation between the shortest and longest inlets. While a strong dependence between mobility and enhancement factor was observed, no correlation was observed between the peptide cross section and enhancement factor (data not shown), which seems to indicate that the major contributor to ion losses in an electrospray capillary inlet is space-charge [16] rather than diffusion.

The results for two different flow rates are also shown in Figure 4 along with the charge state of the peptides. All peaks show an increased improvement in intensity at the lower flow rate, which again suggests that the creation of smaller, more highly charged droplets leads to increased electrospray current loss rates in an inlet capillary. It is also observed that the $3^{+}$charge state of the peptides displays the largest increases in intensity when a shorter inlet is used. The higher charge state results in a higher mobility (see eq 1), which again indicates a bias in the longer inlets against highly charged peptides. This is particularly important in label-free quantitation studies in proteomics when ion intensity is used

Table 1. Peptide species detected by MS in the inlet length study with the corresponding charge state, $m / z$, mobility, and cross-section

\begin{tabular}{lcccc}
\hline \multicolumn{1}{c}{ Peptide } & $\begin{array}{c}\text { Charge } \\
\text { state }\end{array}$ & $m / z$ & $\begin{array}{r}\text { Mobility } \\
\left(\mathrm{cm}^{2} / \mathrm{Vs}\right)\end{array}$ & $\begin{array}{c}\text { Cross-section* } \\
\left(\AA^{2}\right)\end{array}$ \\
\hline \hline Leu-enkephalin & 1 & 556.3 & 0.934 & 227 \\
Bradykinin & 2 & 530.9 & 1.216 & 340 \\
Bradykinin & 3 & 354.3 & 1.352 & 457 \\
Neurotensin & 2 & 837.2 & 1.138 & 369 \\
Neurotensin & 3 & 558.5 & 1.207 & 522 \\
Angiotensin & 2 & 649.0 & 1.092 & 386 \\
Angiotensin & 3 & 433.0 & 1.315 & 473 \\
Angiotensin & 4 & 325.1 & 1.599 & 514 \\
Fibrinopeptide A & 2 & 769.0 & 1.033 & 407 \\
Fibrinopeptide A & 3 & 513.1 & 1.288 & 481 \\
\hline
\end{tabular}

*The mobilities and cross sections for each ion were obtained experimentally by IMS MS in a $\mathrm{N}_{2}$ buffer gas using a $98 \mathrm{~cm}$ IMS drift cell at 4 Torr [23]. 


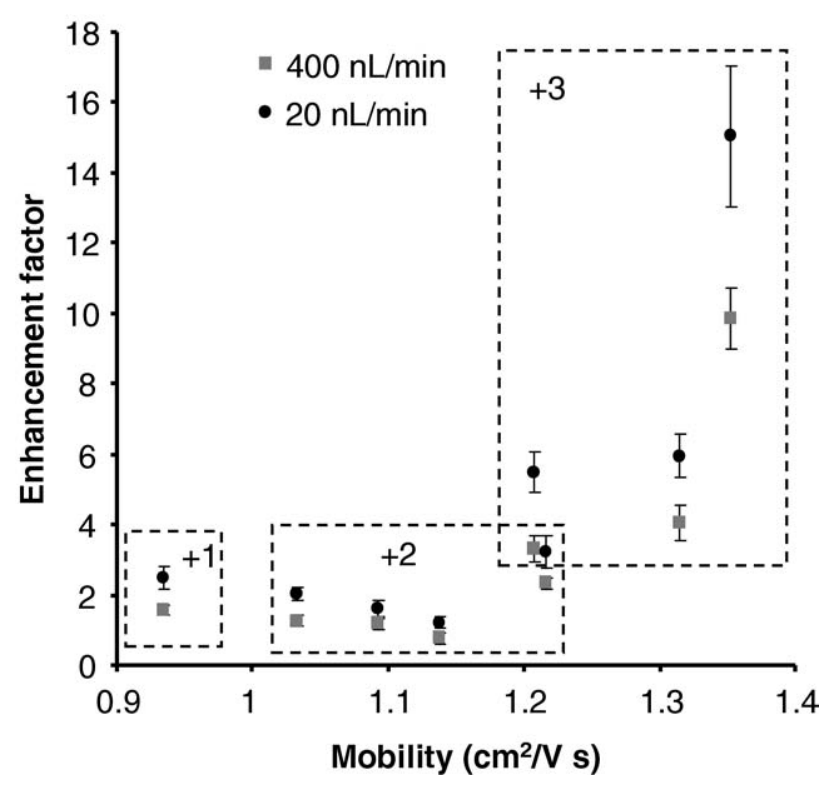

Figure 4. MS signal enhancement factor between the use of a 1.3 $\mathrm{cm}$ inlet and a $6.4 \mathrm{~cm}$ inlet versus mobility for all peptides species detected from a $1 \mu \mathrm{M}$ peptide solution in a LC solvent mixture at two different electrospray flow rates. Charge-state of the peptide is shown by the number and the corresponding box around the data points. Enhancement factor is the ratio of the intensity from using the shortest inlet to the intensity from using the longest inlet.

to determine relative peptide and protein abundances [26]. Use of longer inlets can lead to lower ion intensities for higher charged state species, affecting the MS quantitation.

\section{Inlet Length Effects with a Low-Conductivity Solvent}

Infusion ESI-MS experiments are typically performed with solvents of lower conductivity than those used in LC separations, and the conductivity can have a dramatic effect on the transmission through the standard capillary interface. We have observed that more than $98 \%$ of the total spray current was lost to the front-end of the interface or inside the heated capillary inlet with standard LC solvents [20], compared with $75 \%$ or less when lower conductivity solvents were used [9]. As discussed in the Introduction, the lower conductivity leads to larger droplets with a lower charge density, which should in turn require more desolvation in the inlet to create gas-phase ions. To investigate how inlet length and lower conductivity solvents affect the transmission, we used a common infusion solvent mixture, containing $1 \%$ acetic acid in 50:50 methanol:water. The transmitted electrospray current and inlet transmission efficiency were plotted with respect to inlet length (Supplementary Material Figure S1, which can be found in the electronic version of this article). As in the case of higher conductivity solvents, the electrospray current transmitted into the interface dropped off as the inlet length was increased, 66 to $13 \mathrm{nA}$ from the 1.3 to $6.4 \mathrm{~cm}$ inlets, respectively, at a $400 \mathrm{~nL} / \mathrm{min}$ flow rate. With the shortest inlet, the transmission efficiency reached $\sim 85 \%$ compared with only $\sim 17 \%$ with the high-conductivity solvent. Although the amount of transmitted electrospray current was similar for the two solvent mixtures, the transmission efficiencies differed because the total electrospray current for the infusion solvent was lower $(\sim 70 \mathrm{nA}$ compared with $\sim 380 \mathrm{nA}$ for the LC solvent at $400 \mathrm{~nL} / \mathrm{min}$ flow rate) due to the reduced conductivity of the solution. A greater percentage of the electrospray current was transmitted through the inlet with the infusion solvent due to less space-charge repulsion and larger charged droplets, which reduced the rate of loss inside the inlet.

Figure 5 shows the enhancement factor between the shortest and longest inlet versus peptide mobility. Similar to the previous experiment that used the LC solvent, the increase in intensity is greater for higher mobility species; however, the overall enhancement factor is less significant, and some MS peaks actually display a decrease in intensity when the shortest inlet was used. Since detector response is a combination of transmission and ionization efficiency, the enhancement factors for the infusion solvent compared with the LC solvent suggests that desolvation plays a larger role with the infusion solvent. The tradeoff of desolvation efficiency with transmission efficiency by use of shorter inlets has less of an impact to instrument sensitivity as the gains in transmission efficiency are largely offset by reduced desolvation efficiency. The lower conductivity of the

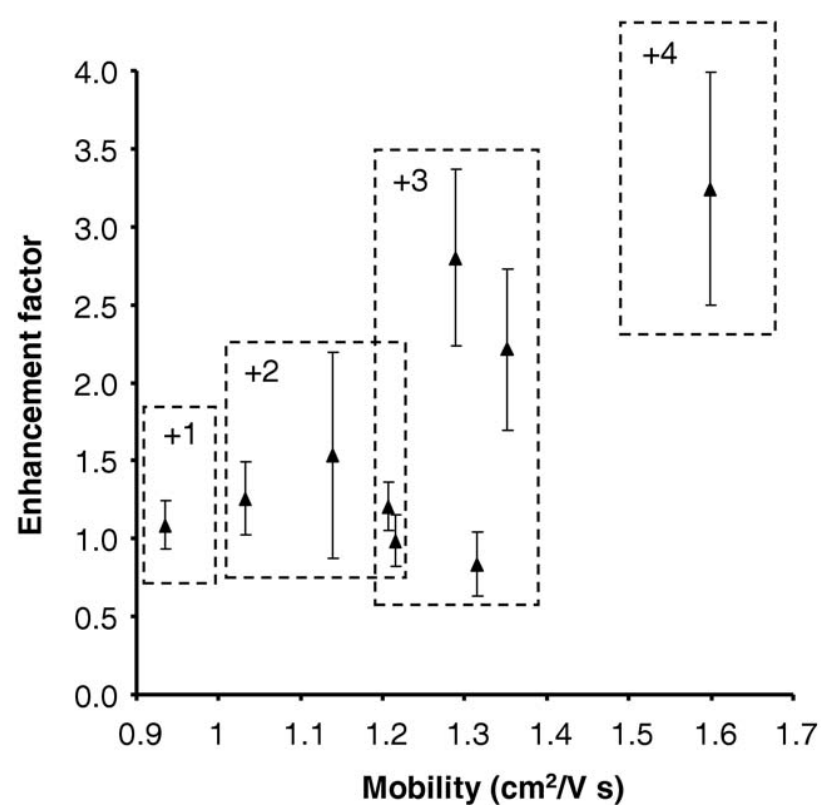

Figure 5. MS signal enhancement factor between the use of a 1.3 $\mathrm{cm}$ inlet and a $6.4 \mathrm{~cm}$ inlet versus mobility for all peptides species detected from a $1 \mu \mathrm{M}$ peptide solution in a methanol:water solvent mixture. Charge-state of the peptide is shown by the number and the corresponding box around the data points. Enhancement factor is the ratio of the intensity from using the shortest inlet to the intensity from using the longest inlet. 
infusion solvent created larger, less charged droplets compared with the LC solvent, and the larger droplets required more desolvation from a longer inlet (increased residence times). In other words, use of the shortest inlet increased the amount of electrospray current entering the interface by $\sim 60 \mathrm{nA}$ for both solvent mixtures, but the lower enhancement factors for the infusion solvent shows that there was less change in the amount of liberated analyte ions even though a larger fraction of the electrospray current was transmitted. A larger portion of the sample was lost due to incomplete desolvation. Importantly, the trend towards higher mobilities and charge states in Figure 5 shows that biases persist even with different solvents that change the interplay between transmission and ionization efficiency with varying inlet lengths.

\section{Temperature and Inlet Length}

Since the gains in transmission efficiency were offset more with the loss in ionization efficiency when shorter inlets were used with the infusion solvent compared with the LC solvent, the 1.3 and $6.3 \mathrm{~cm}$ inlets were evaluated using a range of temperatures. The transmission efficiency of both inlets decreased as the temperature was increased, with the longest inlet having a larger rate of decline. The $1.3 \mathrm{~cm}$ inlet dropped from $91.1 \%$ to $87.8 \%$ when the temperature was increased from 120 to $200{ }^{\circ} \mathrm{C}$, while the $6.4 \mathrm{~cm}$ inlet dropped from $19.8 \%$ to $12.7 \%$. Some of the decrease in ion transmission was due to a decrease in the gas conductance at the higher inlet temperature which was calculated to be $9 \%$ lower. The observed decrease in transmission efficiency for this same temperature increase was 3\% and $36 \%$ for the 1.3 and $6.4 \mathrm{~cm}$ inlets, respectively. This indicates that the change in transmission efficiency for the $6.4 \mathrm{~cm}$ inlet is not due to just the lower gas conductance, but could be from the increased loss rate from increased mobility, diffusion rate, and desolvation/fission events of the electrosprayed droplets. This was evaluated by acquiring mass spectra while varying the temperature of the inlet from 60 to $200{ }^{\circ} \mathrm{C}$. Table 2 shows the resulting MS intensities of three representative peptides normalized to better observe the trend of changing intensity. Not surprisingly, all three peptide intensities increase with increasing temperature as desolvation is improved. However, the rate of increase is varied and corresponds to the peptide mobility. For example, the intensity of $\mathrm{m} / \mathrm{z} 325.1$ for angiotensin $4+$ ions with mobility of 1.599 (see Table 1) increases initially with the increase of the capillary temperature and peaks at $140{ }^{\circ} \mathrm{C}$. Further increase in the capillary temperature resulted in the decrease of the intensity. On the other hand, the intensity of $\mathrm{m} / \mathrm{z} 769.0$ for fibrinopeptide $2+$ ions with mobility of 1.033 continuously increases with the capillary temperature to $200{ }^{\circ} \mathrm{C}$. This indicates that the resulting MS intensity is a combination of increased desolvation efficiency and reduced ion transmission at higher capillary temperature. These two opposing pro-
Table 2. Normalized intensity versus inlet temperature for the $6.4 \mathrm{~cm}$ capillary inlet

\begin{tabular}{|c|c|c|c|c|c|c|}
\hline \multirow[b]{2}{*}{$\begin{array}{c}\text { Temp. } \\
\left({ }^{\circ} \mathrm{C}\right)\end{array}$} & \multicolumn{2}{|c|}{$\mathrm{m} / \mathrm{z} 325.1$} & \multicolumn{2}{|c|}{$m / z 558.5$} & \multicolumn{2}{|c|}{$\mathrm{m} / \mathrm{z} 769.0$} \\
\hline & Ave. & $\begin{array}{c}\% \text { St. } \\
\text { dev. }\end{array}$ & Ave. & $\begin{array}{c}\% \text { St. } \\
\text { dev. }\end{array}$ & Ave. & $\begin{array}{c}\% \text { St. } \\
\text { dev. }\end{array}$ \\
\hline 60 & 1.00 & 8.0 & 1.00 & 21.3 & 1.00 & 25.2 \\
\hline 80 & 1.24 & 10.2 & 1.13 & 14.2 & 1.18 & 13.2 \\
\hline 100 & 1.43 & 5.0 & 1.46 & 10.3 & 1.54 & 11.3 \\
\hline 120 & 1.26 & 18.7 & 1.55 & 4.8 & 1.86 & 7.5 \\
\hline 140 & 1.44 & 3.7 & 1.58 & 3.3 & 1.99 & 6.0 \\
\hline 160 & 1.28 & 16.2 & 1.74 & 7.3 & 2.25 & 1.9 \\
\hline 180 & 1.16 & 17.5 & 1.80 & 3.6 & 2.28 & 2.5 \\
\hline 200 & 0.99 & 13.4 & 1.73 & 1.2 & 2.30 & 3.4 \\
\hline
\end{tabular}

cesses will generally result in an optimum capillary temperature at a given capillary length for each ion species depending on its mobility and diffusion coefficient. A positive intensity increase indicates that ion desolvation is increasing at a greater rate than the ion losses in the inlet, and conversely, if the intensity decreases, the loss rate is greater than the increase in ion desolvation rate. The results in Table 2 suggest that desolvation efficiency improves for all peptides. However, the intensity of angiotensin $4+$ ions reach a maximum at lower temperature $\left(\sim 140^{\circ} \mathrm{C}\right)$ due to a higher mobility than that of fibrinopeptide $2+$ ions.

\section{Conclusions}

With this work, we have sought to provide a basis for improving analytical sensitivity and quantitation when nanoESI is used with MS by investigating the effects of heated capillary inlet length on ionization and transmission efficiency. Even though the experiments were limited to a restricted range of capillary inlet lengths and analytes, the results revealed a complex relationship between charge transmission and analyte desolvation. Changing solution conductivity, flow rate, and inlet temperature affected both transmission and desolvation efficiencies, highlighting the interplay between transmission and ionization efficiency. We showed that the signal response to the interface geometry and conditions is far from universal; even with a relatively simple sample of only five peptides, some species showed only a small improvement with decreasing inlet length, while other species showed greater than 10 -fold increase. This pointed to suppression of certain species (e.g., higher mobility analytes) when longer inlets were used. Longer capillary inlets could be a used as a means of suppressing signals from lower $\mathrm{m} / \mathrm{z}$ chemical background species [27], but this could also lead to a bias against, e.g., higher charge states of peptides.

Overall, the results show that longer capillary inlets can lead to biases in the electrospray current transmission, even when analyzing relatively simple solutions. A more comprehensive study is needed utilizing a broader range of chemical species so as to better sepa- 
rate the complex interplay of desolvation and transmission efficiency and isolate ion losses inside the capillary inlet. While clearly, other factors in this study also can contribute to the bias, the results shown here can be used to aid in the design of ESI-MS interfaces and in operating the electrosprays to improve absolute quantitation in MS measurements.

\section{Acknowledgments}

The authors acknowledge support for portions of this research by the NIH National Center for Research Resources (RR018522), the NIH National Cancer Institute (R21 CA126191), and the National Institute of Allergy and Infectious Diseases NIH/DHHS through interagency agreement Y1-AI-4894-01. Experimental portions of this research were performed in the Environmental Molecular Sciences Laboratory, a U.S. DOE national scientific user facility located at the Pacific Northwest National Laboratory (PNNL) in Richland, Washington. PNNL is a multiprogram national laboratory operated by Battelle for the DOE under contract no. DEAC05-76RLO 1830.

\section{Appendix A Supplementary Material}

Supplementary material associated with this article may be found in the online version at doi:10.1016/ j.jasms.2009.08.018.

\section{References}

1. El-Faramawy, A.; Siu, K. W. M.; Thomson, B. A. Efficiency of NanoElectrospray Ionization. J. Am. Soc. Mass Spectrom. 2005, 16, 1702-1707.

2. Schmidt, A.; Karas, M.; Dulcks, T. Effect of Different Solution Flow Rates on Analyte Ion Signals in Nano-ESI MS, or: When Does ESI Turn into Nano-ESI? J. Am. Soc. Mass Spectrom. 2003, 14, 492-500.

3. Covey, T. R.; Thomson, B. A.; Schneider, B. B. Atmospheric Pressure Ion Sources. Mass Spectrom. Rev. 2009, DOI 10.1002/mas. 20246.

4. Kebarle, P.; Verkerk, U. H. Electrospray: From Ions in Solution to Ions in the Gas Phase, What We Know Now. Mass Spectrom. Rev. 2009, DOI 10.1002/mas. 20247

5. Gale, D. C.; Smith, R. D. Small Volume and Low Flow-Rate Electrospray Ionization Mass Spectrometry of Aqueous Samples. Rapid Commun. Mass Spectrom. 1993, 7, 1017-1021.

6. Wilm, M. S.; Mann, M. Electrospray and Taylor-Cone Theory, Dole's Beam of Macromolecules at Last? Int. J. Mass Spectrom. Ion Processes 1994, 136, 167-180.

7. Wilm, M.; Mann, M. Analytical Properties of the Nanoelectrospray Ion Source. Anal. Chem. 1996, 68, 1-8.

8. Fernandez de la Mora, J.; Loscertales, I. The Current Emitted by Highly Conducting Taylor Cones. J. Fluid Mech. 1994, 260, 155-184.
9. Page, J. S.; Kelly, R. T.; Tang, K.; Smith, R. D. Ionization and Transmission Efficiency in an Electrospray Ionization-Mass Spectrometry Interface. J. Am. Soc. Mass Spectrom. 2007, 18, 1582-1590.

10. Emmett, M. R.; White, F. M.; Hendrickson, C. L.; Shi, S. D.-H.; Marshall, A. G. Application of Micro-Electrospray Liquid Chromatography Techniques to FT-ICR MS to Enable High-Sensitivity Biological Analysis. J. Am. Soc. Mass Spectrom. 1998, 9, 333-340.

11. Shen, Y.; Tolic, N.; Masselon, C.; Pasa-Tolic, L.; Camp, D. G.; Hixson, K. K.; Zhao, R.; Anderson, G. A.; Smith, R. D. Ultrasensitive Proteomics Using High-Efficiency On-Line Micro-SPE-NanoLC-NanoESI MS and MS/MS. Anal. Chem. 2004, 76, 144-154.

12. Shen, Y.; Tolic, N.; Masselon, C.; Pasa-Tolic, L.; Camp, D. G.; Lipton, M. S.; Anderson, G. A.; Smith, R. D. Nanoscale Proteomics. Anal. Bioanal. Chem. 2004, 378, 1037-1045.

13. Electrospray Ionization Mass Spectrometry: Fundamentals, Instrumentation, and Applications; John Wiley and Sons, Inc.: New York, 1997; pp 107-136.

14. Chowdhury, S. K.; Katta, V.; Chait, B. T. An Electrospray-Ionization Mass Spectrometer with New Features. Rapid Commun. Mass Spectrom. 1990, 4, 81-87.

15. Busman, M.; Sunner, J.; Vogel, C. R. Space-Charge-Dominated MassSpectrometry Ion Sources-Modeling and Sensitivity. J. Am. Soc. Mass Spectrom. 1991, 2, 1-10.

16. Lin, B. W.; Sunner, J. Ion Transport by Viscous Gas Flow Through Capillaries. J. Am. Soc. Mass Spectrom. 1994, 5, 873-885.

17. Page, J. S.; Tolmachev, A. V.; Tang, K.; Smith, R. D. Theoretical and Experimental Evaluation of the Low $\mathrm{m} / \mathrm{z}$ Transmission of an Electrodynamic Ion Funnel. J. Am. Soc. Mass Spectrom. 2006, 17, 586-592.

18. Kelly, R. T.; Page, J. S.; Luo, Q.; Moore, R. J.; Orton, D. J.; Tang, K.; Smith, R. D. Chemically Etched Open Tubular and Monolithic Emitters for Nanoelectrospray Ionization Mass Spectrometry. Anal. Chem. 2006, 78, 7796-7801.

19. Tomlinson, E.; Jefferies, T. M.; Riley, C. M. Ion-Pair High-Performance Liquid Chromatography. J. Chromatogr. 1978, 159, 315-358.

20. Marginean, I.; Kelly, R. T.; Prior, D. C.; LaMarche, B. L.; Tang, K.; Smith, R. D. Analytical Characterization of the Electrospray Ion Source in the Nanoflow Regime. Anal. Chem. 2008, 80, 6573-6579.

21. Wutz, M.; Adam, H.; Walcher, W. Theory and Practice of Vacuum Technology; Vieweg: Braunschweig, 1989; p. 96.

22. Mason, E. A.; McDaniel, E. W. Transport Properties of Ions in Gases; Wiley: New York, 1988; pp 77-78.

23. Baker, E. S.; Clowers, B. H.; Li, F. M.; Tang, K.; Tolmachev, A. V.; Prior D. C.; Belov, M. E.; Smith, R. D. Ion Mobility Spectrometry-Mass Spectrometry Performance Using Electrodynamic Ion Funnels and Elevated Drift Gas Pressures. J. Am. Soc. Mass Spectrom. 2007, 18, 1176-1187.

24. Henderson, S. C.; Valentine, S. J.; Counterman, A. E.; Clemmer, D. E. ESI/Ion Trap/Ion Mobility/Time-of-Flight Mass Spectrometry for Rapid and Sensitive Analysis of Biomolecular Mixtures. Anal. Chem. 1999, 71, 291-301.

25. Batchelor, G. K. An Introduction to Fluid Dynamics; Cambridge University Press: Cambridge, 1967; p. 233.

26. Yang, F.; Jaitly, N.; Jayachandran, H.; Luo, Q.; Monroe, M. E.; Du, X.; Gritsenko, M. A.; Zhang, R.; Anderson, D. J.; Purvine, S. O.; Adkins, J. N.; Moore, R. J.; Mottaz, H. M.; Ding, S.-J.; Lipton, M. S.; Camp, D. G.; Udseth, H. R.; Smith, R. D.; Rossie, S. Applying a Targeted Label-Free Approach Using LC MS AMT Tags to Evaluate Changes in Protein Phosphorylation Following Phosphatase Inhibition. J. Proteome Res. 2007, 6, 4489-4497.

27. Guo, X.; Bruins, A. P.; Covey, T. R. Method to Reduce Chemical Background Interference in Atmospheric Pressure Ionization Liquid Chromatography Mass Spectrometry Using Exclusive Reactions with the Chemical Reagent Dimethyl Disulfide. Anal. Chem. 2007, 79, 40134021. 